\title{
Professionnalisation d'une formation d'ingénieurs par la mise en ouvre d'une pédagogie active associant le monde industriel
}

\author{
Marc Pasquet [marc.pasquet@ensicaen.fr] \\ Sylvain Vernois [sylvain.vernois@ensicaen.fr] \\ ENSICAEN, 6 Boulevard du Maréchal Juin, 14050 CAEN cedex 4
}

\begin{abstract}
Résumé
L'objectif de cet article est de présenter l'expérimentation d'une pédagogie active dans une formation d'ingénieurs de l'ENSICAEN, en s'appuyant la mise en œuvre d'une nouvelle plateforme pédagogique professionnelle définie avec les entreprises partenaires. L'originalité de cette plateforme est qu'elle n'existe que pour et par les élèves : ils en sont non seulement les utilisateurs, mais aussi les constructeurs grâce aux nombreux projets qu'ils réalisent.
\end{abstract}

Mots clés : formation d'ingénieurs, plateforme pédagogique, plateforme industrielle, monétique

\section{OBJECTIFS ET FINALITES}

Depuis 1994, l'ENSICAEN forme des ingénieurs en informatique spécialisés dans les domaines de la Monétique et la Sécurité des Systèmes. La monétique peut se définir comme «l'ensemble des techniques électroniques, informatiques et télématiques permettant d'effectuer des transactions, des transferts de fonds ou toute autre opération qui relie un utilisateur final équipé d'une carte avec un ensemble de services ».

Jusqu'en 2002, les méthodes d'enseignement de cette option étaient organisées de façon traditionnelle autour de séances de Cours, de Travaux Dirigés et de Travaux Pratiques. Des outils spécifiquement acquis permettaient aux élèves de mettre en pratique les connaissances acquises en cours :

- TP de sécurité informatique

- TP d'utilisation d'un simulateur de réseau bancaire

- $\quad$ TP de communication et exploitation de cartes à puce..

Cependant, aucun TP ne pouvait être mis en œuvre impliquant l'intégralité de la chaîne monétique, les outils en place n'étant pas liés entre eux.

En 2002, à la demande de ses partenaires industriels, l'ENSICAEN a décidé de préparer en complément de la formation existante, une nouvelle formation par apprentissage «ingénieur informatique option monétique et sécurité des systèmes ». L'aspect professionnalisant de l'apprentissage a été l'occasion de revoir les méthodes pédagogiques, en particuliers celles liées à l'option, afin de former des ingénieurs plus opérationnels, mieux formés et en phase avec les attentes de leurs futurs employeurs, dès leur sortie de l'école.

C'est ainsi qu'il est apparu très vite nécessaire de mettre en œuvre une plateforme monétique pédagogique, composée de systèmes industriels performants, afin de constituer un outil complémentaire de formation de très haut niveau à même de générer et pérenniser une activité de formation et de recherche sur les aspects matériels et logiciels de la monétique. Cette plateforme bancaire complète, qui permettra de réaliser des transactions de retrait et de paiement de bout en bout, constitue une première mondiale dans le domaine de la formation.

Le projet de mise en œuvre de cette plateforme a démarré en 2004, avec comme envie d'associer les industriels, l'équipe enseignante et les élèves de l'école dès la création.

\section{AUtOUR de la Plateforme}

\subsection{Une équipe d'enseignants spécialisés}

Acquérir une plateforme monétique industrielle signifiait pour l'école de disposer d'une connaissance précise des processus et fonctions mis en œuvre, à l'image d'un organisme bancaire.

C'est pourquoi l'un des points importants a été le recrutement en renfort par l'ENSICAEN de personnes issues du monde industriel monétique. Ainsi, la capacité d'enseignement de cette option en direction des élèves s'est renforcée et professionnalisée. Dans le même temps, l'équipe chargée de la mise en place et du suivi ultérieur de la plateforme est née.

\subsection{Les étapes}

L'identification des fonctions présentes dans une chaîne monétique n'est pas chose aisée, mais surtout l'identification des services attendus sur la plateforme pédagogique. 
C'est pourquoi le projet s'est finalement décomposé en 2 grandes phases :

- Mise en place des briques de base pour assurer la chaîne minimale,

- Ajout d'extensions et nouvelles possibilités afin d'aller plus loin dans les expérimentations au fur et à mesure des besoins.

\subsubsection{Phase 1}

La phase 1 a consisté à créer une plateforme constituée de 2 banques capables d'échanger des transactions monétiques au travers de réseaux domestiques ou internationaux. Ces 2 banques sont équipées de $\mathrm{GAB}^{1}$ et de $\mathrm{TPE}^{2}$ et sont toutes les deux capable d'émettre des cartes bancaires $\mathrm{EMV}^{3}$. Au-delà de la mise en place technique, les aspects sécuritaires ont été traités avec toutes les contraintes imposées à une entreprise du domaine. A titre d'exemple, il a été étudié et géré le fait que les cartes «bancaires » émises dans le cadre du projet ne puissent être utilisées sur les distributeurs et les TPE en dehors de ceux dédiés de l'ENSICAEN.

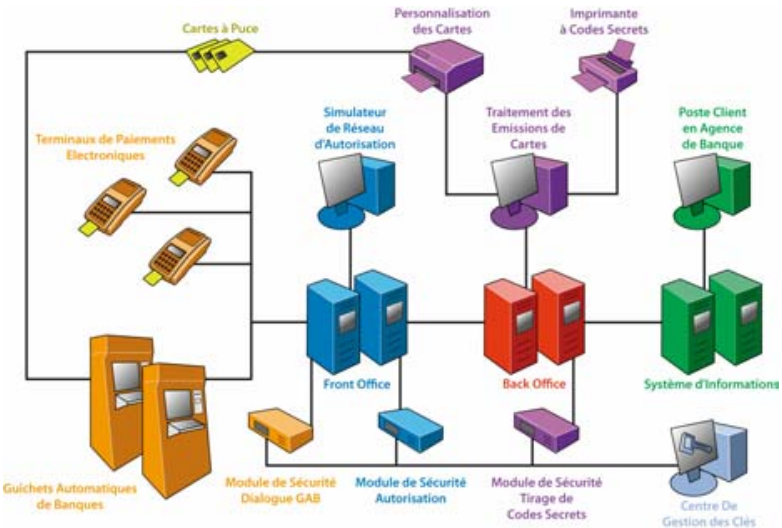

Figure 1 : Schéma de la plateforme en phase 1

Les premières briques mises en place sont :

- Les cartes de type bancaire,

- Les terminaux (TPE, GAB...),

- Les fonctions de front-office,

- Les fonctions de back-office,

- Les réseaux d'interconnexion bancaires,

- Les éléments de sécurité.

Chaque brique a été prise en charge par un enseignant de l'école, devenu de fait le contact privilégié avec la ou les entreprises fournissant la brique. Ainsi, des relations privilégiées ont pu être développées entre l'école et les industriels, facilitant à la fois les discussions et la transmission de la connaissance vers les enseignants et les élèves.

\footnotetext{
${ }^{1}$ GAB : Guichet Automatique de Banque

2 TPE: Terminaux de Paiement Electronique, utilisés chez les commerçants

${ }^{3}$ EMV : Eurocard Mastercard Visa : spécifications internationales visant à disposer de cartes bancaires compatibles au niveau international.
}

\subsubsection{Phase 2}

La phase suivante consistera en l'ajout de fonctionnalités, qui permettront non seulement de maintenir à niveau la plateforme par rapport à la réalité du terrain, mais également et surtout de s'intéresser à des sujets nouveaux et ainsi prendre de l'avance sur les déploiements.

Formés sur un tel environnement, les ingénieurs auront un pas d'avance sur le marché, et seront plus à même de faire évoluer les systèmes.

\section{VERS UNE PEDAGOGIE PAR PROJET}

L'intégration des élèves au projet est un point qui fait partie du projet plateforme lui-même. En effet, la PMP est par nature dédiée à la pédagogie, il nous a donc paru indispensable d'y associer au plus tôt les futurs « utilisateurs ».

La répartition des rôles s'est organisée de la manière suivante :

- Les entreprises apportent leurs outils sur la plateforme, et leurs compétences qu'ils diffusent aux élèves et enseignants, sous forme de formations de type professionnel,

- Les élèves reçoivent les formations, et exploitent leurs connaissances en participant de façon active à la mise en place de la plateforme,

- L'équipe enseignante joue le rôle de coordinateur du projet et de tuteur des élèves.

Si le rôle de l'entreprise dans le projet plateforme était évident dès le début, les rôles des élèves et des enseignants ne sont apparus qu'en cours de projet, suite aux états d'avancement réalisés: une pédagogie par projet avait trouvé sa place de façon naturelle, aussi bien parmi les enseignants que les élèves.

Avant même la finalisation du projet phase 1, une douzaine d'élèves ingénieurs de $2^{\text {ème }}$ année ont participé pendant 2 mois, sous tutorat des équipes enseignantes, aux différentes études préliminaires :

- Etude des problématiques sécuritaires liées à un environnement totalement conforme aux normes bancaires, mais dont l'usage ne doit en aucun cas pouvoir interférer avec les environnements en production;

- Etudes techniques et fonctionnelles des interfaçages à développer entre les briques acquises par l'école, allant parfois jusqu'à la réalisation de l'interface ;

- $\quad$ Etude des besoins d'un système d'information bancaire, réalisation du cahier des charges et développement du SI.

Ces problématiques, difficilement abordables lors de séances de cours ou de Travaux Pratiques traditionnelles, ont permis aux élèves d'être confrontés à des situations réelles. Tout d'abord désorientés devant l'étendue des sujets proposés, ils ont peu à peu avancé, en posant des questions ou en 
recherchant eux même les informations manquantes parmi celles mises à disposition par les entreprises.

L'existence même de cette plateforme a ouvert naturellement la porte à l'introduction de manipulations nouvelles et originales pour les élèves ingénieurs.

Au-delà des Travaux Pratiques, c'est la façon d'enseigner qui peut évoluer, et être au plus près du besoin des entreprises.

Disponible depuis la rentrée 2006, la plateforme a déjà été exploitée par les apprentis ingénieurs de $3^{\text {ème }}$ de l'ENSICAEN. 13 d'entre eux ont disposé de 14 jours chacun pour réaliser des projets, complémentaires aux enseignements, et surtout prétextes à aborder des sujets et problèmes concrets et complexes. Suivis tout du long par l'équipe enseignante, les élèves ont été de véritables acteurs de leur formation, en progressant au fur et à mesure de leurs questions. Les projets ainsi réalisés sont venus compléter la plateforme existante.

\section{UNE PLATEFORME VIVANTE}

\subsection{Des technologies et des services qui} évoluent

La concrétisation de la plateforme pédagogique ne doit pas être considérée comme une fin au projet. En effet, si elle est aujourd'hui conforme à la réalité industrielle, et est un outil pédagogique de très grande valeur, elle risque de devenir rapidement obsolète avec les évolutions technologiques et de services inhérents au marché. C'est pourquoi une veille permanente doit être mise en place, en s'appuyant sur les futurs besoins identifiés par les partenaires de la plateforme, mais aussi en prenant en compte les progrès réalisés par la recherche universitaire dans les domaines connexes.

Cette veille peut être réalisée par les spécialistes de l'école, mais aussi et surtout dans le cadre de projets d'élèves.

Six grandes évolutions sont aujourd'hui identifiées, et feront l'objet de projets qui, petit à petit, seront intégrés à la plateforme par les promotions d'élèves à venir.

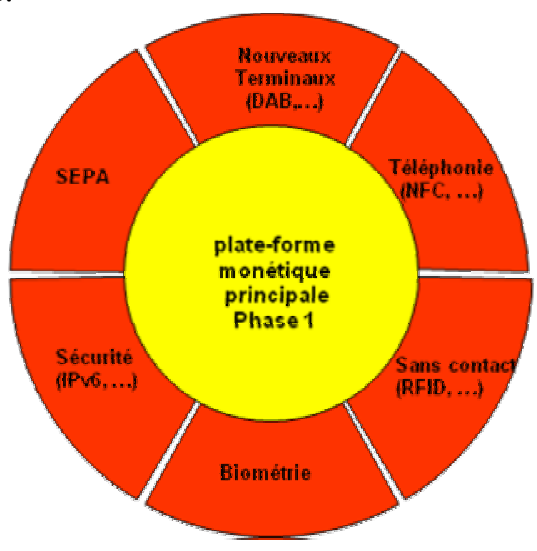

Figure 2 : La plateforme en phase 2

\subsubsection{Nouveaux terminaux}

Si les terminaux bancaires ont peu évolué au cours des 10 dernières années, cette évolution s'est accélérée et implique maintenant l'ensemble des terminaux de paiement :

- Les terminaux traditionnels doivent évoluer pour :

- s'adapter aux nouveaux services (paiement de facture sur les GAB, rechargement de GSM sur les TPE, ...)

- s'intégrer dans les nouveaux réseaux (IPV6 pour les GAB, ADSL pour les TPE, GSM, ...)

- permettre l'authentification biométrique

- Des lecteurs sont installés sur les PC des particuliers pour la sécurisation des transactions à distance,

- Des téléphones mobiles deviennent des terminaux de paiement qu'il va falloir adapter en conséquence,

- Les terminaux de paiement sans contact sont en expérimentation.

\subsubsection{Applications RFID}

Avec l'apparition des cartes sans contact, deux marchés sont en train de s'unir : celui de la carte à puce à contact et celui de l'identification par radiofréquence de type RFID $^{4}$. Les systèmes RFID ont, à l'origine, été développés pour l'identification sans contact d'objets. Ces systèmes ont peu à peu été découverts pour l'automatisation de processus dans l'industrie, et en plus d'une fonction de lecture, une fonction d'écriture a été développée en vu de fournir à l'utilisateur une possibilité de stockage d'informations.

Les paiements sans contact sont beaucoup plus pratiques pour les consommateurs et sont particulièrement adaptés dans des environnements d'achat où la rapidité est essentielle, comme la restauration rapide, les stations-service, les petits commerces et supermarchés, mais aussi les cinémas. Ils offrent également des opportunités nouvelles de paiement par carte dans des environnements d'achat inhabituels, comme les distributeurs automatiques et les péages.

\subsubsection{Technologie NFC}

La technologie de communication $\mathrm{NFC}^{5}$ embarquée dans les téléphones mobiles concrétise le mariage du sans fil et du sans contact. Elle transforme les mobiles en communicateurs universels susceptibles de dialoguer avec leur environnement immédiat. Grâce au sans contact, les mobiles permettent d'échanger des données et de réaliser des transactions avec une grande facilité d'usage. Le mobile devient ainsi titre de transport, badge d'accès, moyen de paiement... Ce mariage annonce

\footnotetext{
${ }^{4}$ RFID : Radio Frequency Identification

${ }^{5}$ NFC : Near Field Communication
} 
le déploiement d'une vaste gamme d'applications inédites.

A l'horizon 2010 plus de $50 \%$ des téléphones mobiles (soit plus de 500 millions) devraient être équipés d'une puce NFC.

\subsubsection{Biométrie}

L'accroissement des capacités de stockage et de calcul permet d'envisager le traitement de données biométriques par une simple puce, placée sur une carte ou une clé USB. Associée au sans contact, on peut imaginer un recours croissant à la biométrie pour l'authentification des individus : contrôle d'accès locaux ou réseau, titre d'identité électronique, ...).

Si elle présente une commodité d'usage certaine (plus besoin de se souvenir de multiples mots de passe, l'individu porte la clé unique en lui-même), la biométrie pose des problèmes d'éthique et de sécurité qui suscitent la défiance car elle renvoie l'individu à une identité physique unique et fait naître des craintes quant à la protection de la vie privée, la sécurisation des données enregistrées et la fiabilité des systèmes.

\subsubsection{Sécurité}

En monétique, l'ensemble de la cryptographie est délégué à des systèmes externes qui se présentent sous forme de boîtiers protégés et équipés de logiciels non accessibles que l'on appelle des $\mathrm{HSM}^{6}$. Ces enceintes sécurisées ne sont pas modifiables autrement que par le constructeur et n'évoluent qu'à la suite de préconisations normatives ou règlementaires. Dans le cas d'expérimentations pré normatives sur la partie sécurité de la chaîne monétique, cette limitation s'avère être problématique. Les entreprises spécialisées se proposent de nous fournir des HSM dont une partie au moins du logiciel pourrait être modifiable en langage JAVA afin d'expérimenter sur la plate-forme monétique :

- Des architectures de sécurité normalisées dans le cadre d'autres usages que la monétique (Passeport électronique, signature électronique, ...)

- De nouveaux algorithmes de chiffrement afin d'améliorer la sécurité de la monétique dans un futur proche (AES, Courbes Elliptiques, Cryptographie Quantique, ...)

\subsubsection{Evolutions réglementaires}

Le SEPA (Single European Payment Area) est un projet instauré par l'EPC ${ }^{7}$ (Conseil des Paiements Européens) et la banque centrale européenne $\left(\mathrm{ECB}^{8}\right)$.

Le SEPA a pour but de mettre en place une architecture de paiement permettant à tous les

\footnotetext{
${ }^{6}$ HSM : Hardware Security Module

${ }^{7}$ EPC : European Payments Council

${ }^{8}$ ECB : European Central Bank
}

organismes et particuliers de la zone unifiée européenne d'effectuer des paiements au sein de cette zone avec tous les avantages d'un paiement domestique (rapidité, faible coût et sécurité de la transaction). Cette initiative a été motivée dans un but économique, en effet, les forts coûts des transactions transfrontalières européennes constituaient un frein au développement du marché européen de la banque. Et en particulier, ne permettait pas une concurrence libre et non faussée telle que l'Europe la préconise. A terme, il n'y aura plus de distinction entre les paiements nationaux et les paiements transfrontaliers. Le programme de travail se déroulera du 1er Janvier 2008 au 31 décembre 2010, date à laquelle SEPA sera déployée sur toutes l'Europe.

\section{BILAN}

Au-delà de la simple mise en œuvre technique, la Plateforme Monétique Pédagogique est la démonstration qu'entreprises et écoles ont tout intérêt à se rapprocher l'une de l'autre :

- L'école peut y gagner un formidable outil permettant de faire évoluer la pédagogie en impliquant complètement les élèves dans leur formation et en les rendant acteurs dans l'évolution de l'outil lui-même.

- Les entreprises, en échange d'un investissement en matériel, logiciel et formation, peuvent prendre une part active à la formation des élèves et s'assurer un vivier de futurs diplômés à la pointe du domaine chaque année.

\section{POUR PLUS D'INFORMATION...}

- $\quad$ http://www.monetique.ensicaen.fr/ 